\title{
Flying Height Optimization for Unmanned Aerial Vehicles in Cellular - Flying Adhoc NETWORK
}

\author{
Quynh Tu NGO*, Duc Ngoc Minh DANG, Khoa Anh TRAN
}

Faculty of Electrical and Electronics Engineering, Ton Duc Thang University, Ho Chi Minh City, Vietnam

*Corresponding Author: Quynh Tu NGO (email: ngotuquynh@tdtu.edu.vn)

(Received: 15-October-2018; accepted: 06-December-2018; published: 31-December-2018)

DOI: http://dx.doi.org/10.25073/jaec.201824.210

\begin{abstract}
In Flying Adhoc NETwork (FANET), the communications between Unmanned Aerial Vehicles (UAV), UAVs to infrastructure, and UAVs to wireless sensors are crucial design factors. With strict energy constrain during flying operation, the allocation of power and resources, the flying strategy, and the medium access mechanism shall be effectively used. When employing cellular network as backhaul for UAVs, the flying height of UAVs not only affects the communication between UAVs and end users on the ground, but also determines the reception between infrastructure (base stations from the cellular network) to UAVs. In this paper, we optimize the flying height of UAVs for better reception from the cellular network by using stochastic geometry analysis to model the aggregated interference at the UAV side. The network system performance is also examined under the effect of fading-less channel and Rayleigh fading channel.
\end{abstract}

\section{Keywords}

FANET, UAV, Flying Height, Optimization.

\section{INTRODUCTION}

Flying Adhoc NETwork (FANET) is an adhoc network connecting autonomous flying vehicles, which are referred to as the Unmanned Aerial Vehicles (UAVs). With the development of the Internet of Things, employing UAV in the wireless communication process has attracted notable attention. With some unique features like changing topology, fluid with number of UAVs, changing link and changing relative position of UAVs, FANET has brought a lot of challenges in the design of PHY/MAC. With limited battery capacity, some UAVs might be offline from the network due to malfunction or power lost which makes the network topology changes more frequent. As a result, the network link can also be formed and vanished with respect to the change in position of UAVs.

The cellular-flying adhoc network refers to two communication networks: the communication between cellular infrastructure to UAVs where UAVs are clients, and the communication between UAVs to end users on the ground where UAVs act as servers. To deliver reliable services, UAVs in the network not only have their optimal connectivity maintain for end users, but also have to get adequate reception from the cellular network. The first aforementioned 
type of communication between UAVs and end users has drawn more attention since there has been an amount of works on modeling the network and optimizing the position of UAVs (two-dimension position) for better coverage to end users. This paper focuses on optimizing the third dimension of UAVs' position, the UAVs' flying height, for better reception from the cellular network.

In this paper, Poisson Point Process has been used to model the location distribution of cellular base stations. The system performance is analyzed with the help of stochastic geometry. The purpose of cellular - FANET is employing the cellular reception onto UAVs so that UAVs could offer coverage for end users on the ground. A successful cellular reception is counted when the Signal-to-Interference-plus-Noise-Ratio is greater than the threshold at UAV antenna. By computing the aggregated interference at UAV side, the probability of getting successful cellular reception is evaluated in this paper. The flying height optimization problem is done with the desire variable is the random variable representing the real distance between UAV and base station. The objective function in this optimization problem is the probability of getting successful reception from base station at UAV side. From the analytical results, insight on factors that effect the network performance are also discussed.

The remains of this paper are organized as follows: Section 2 is the literature review on UAV networks modeling. Section 3 describes the system model in detail, the mathematical analysis is in Section 4. Section 5 is on evaluating the system performance through simulation and numerical results, and the paper is concluded in Section 6.

\section{RELATED WORKS}

Works have been done on unmanned aerial vehicle networks can be categorized as follows:

- Channel modeling
- Performance analysis

- Trajectory optimization

- Cellular connected UAV.

In the communication between UAVs and end users on the ground, authors in [1 model the air-to-ground path-loss for low altitude platforms in the urban environment. The work in 2 derives an optimal UAV altitude function for maximum coverage on the ground in the urban environment. A closed form formula for predicting the probability of line-of-sight between UAV and end users is also presented in 2. For high altitude platforms, path-loss and fading for air-ground link has been modeled in [3. A comprehensive review on air-ground channel modeling is presented in 4 with the challenges considered for unmanned aircraft systems. The empirical channel model is done in 5] for the unmanned ground vehicle (including sensor network), which is considered as end users on the ground in UAV network. The work in 5 can be adopted into cluster sensor network, then cluster heads communicate to UAVs. An air-ground channel selection method using digital elevation model data is proposed in [6]. The model is derived based on the measurement results for urban and suburban environment. Authors in [7 introduce a geometry-based stochastic model for UAV channel modeling and propose a novel three dimensional geometry-based stochastic model for UAV - MIMO channel.

In the category of analyzing the network performance, a use case for UAV as flying base station is analyzed in [8. A framework coverage and rate analysis are derived for the downlink data transmission and an underlaid device-to-device communication network 8]. The optimal dimensioning and performance analysis for drone-based wireless communications are also presented in [9]. The work extends the traditional models with considering the transmitter antenna gain patterns and multipath fading.

UAV trajectory is one of the key parameters in UAV-end user communications. Optimizing 
UAV trajectory to enhance the network performance is done in [10] and 11]. The work in [10 applies the block coordinate descent and successive convex optimization to optimize the multiuser communication scheduling and association jointly with UAV trajectory and power control for UAV-end user network. [11. presents an efficiently joint transmit power and trajectory optimization algorithm. The work results in water-filling characteristic in spatial domain for the optimized transmit power.

Employing cellular network in assisting UAV connection, the work in [12] analyzes the use of LTE in transferring large amount of data from UAV to the ground. The impact of interference and path-loss on data transmission to and from UAV have also been studied. An in-depth analysis on the coverage probability of cellular network for aerial and ground users has been done in [13. Modeling the channel with Nakagami-m fading model, [13] evaluates the base station height effect on the coverage probability. Authors in [14 identify the typical airborne connectivity requirements and characteristics of the LTE connectivity for low altitude small UAVs. Different propagation conditions are also highlighted with measurement and ray tracing results.

The main difference of this paper to the above works is that the UAV flying height being optimized through the analysis of the signal-tointerference-plus-noise-ratio under various base station density scenarios with the present of fading.

\section{NETWORK MODEL}

For different wireless technologies, to achieve the expected accuracy in estimating the network performance, system level simulation is required and needs to be implemented on a specific geometrical environment. In addition, to confirm the accuracy of the simulation, analytical approach is also required. The analytical approach could give an insight into the factors that affect the network performance.
In cellular-FANET, the Base Station (BS) density, the channel model and the resource allocation all have direct effect to the network performance. To model the location of BSs, stochastic geometry has been widely used.

Assuming that the BS location follows homogeneous Poisson Point Process (PPP), with $\lambda$ BSs per unit area, the distribution of BSs is denoted as

$$
\Phi=\left\{X_{n} \in \mathbb{R}^{2}\right\}_{n \in \mathbb{N}} .
$$

Defining serving BS for an UAV is the BS that UAV gets the highest received power, average cellular boundary is the region where all UAVs are associated to a serving BS. The average cellular boundary of each BS $X_{n}$ is the Voronoi cell $V\left(X_{n}\right): V\left(X_{n}\right):=$ $\left\{q \in \mathbb{R}^{2} \mid\left\|q-X_{n}\right\| \leq\left\|q-X_{i}\right\| \forall X_{i} \in \Phi \backslash\left\{X_{n}\right\}\right\}$ with $\Phi$ is the BS set. The network is considered as a homogeneous network with all BSs are assumed to continuously transmit a constant power level of $P_{o}$. The UAVs distribution is also considered a homogeneous distribution.

The received power at a certain UAV has encounter some power losses with respect to the BS transmitted power. These power losses mainly include path-loss and fading, where both slow fading (shadowing effect) and fast fading (Rayleigh fading for non-line-of-sight signals and Rician fading for line-of-sight signals) are present. The received power at a UAV is denoted as:

$$
P_{R X}=P_{o} . \text { g.h.L, }
$$

where $g$ is a random variable for log-normal shadowing, $h$ is a random variable represents fast fading, $L$ is the path-loss.

- The log-normal shadowing:

$$
g=\exp (Y),
$$

where $Y$ is normally distributed with mean $\mu$ and standard deviation $\frac{\ln (10)}{10} \sigma_{d B}$, where $\sigma_{d B}$ is the standard deviation in dB. $\sigma_{d B}$ usually ranges from $4 \mathrm{~dB}$ to $13 \mathrm{~dB}$ for outdoor channels [15].

- The cellular to UAV path-loss includes terrestrial path-loss and the aerial excess pathloss, where the aerial excess path-loss model 


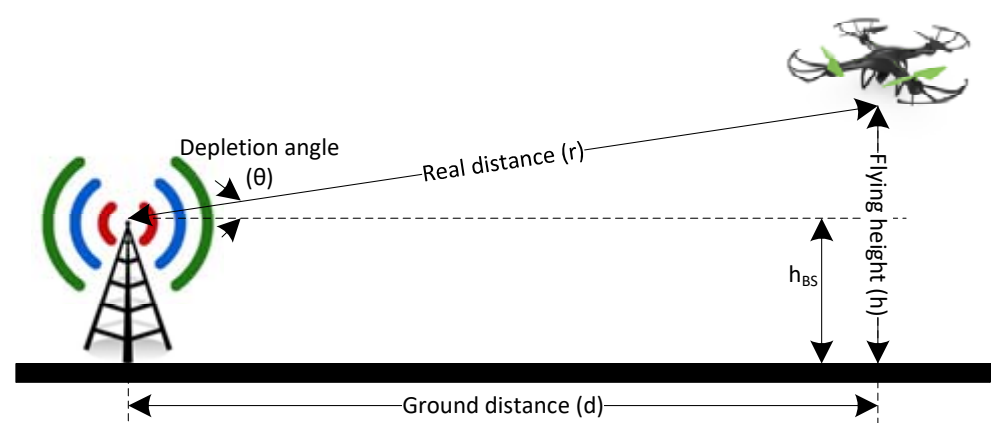

Fig. 1: Serving base station to UAV channel with base station height of $h_{B S}$

is done for suburban environment in [16. The cellular to UAV path-loss is as below:

$$
\begin{aligned}
L(d, \theta) & =10 \alpha \log (d) \\
& +A\left(\theta-\theta_{o}\right) \exp \left(-\frac{\theta-\theta_{o}}{B}\right) \\
& +\eta_{o}+\mathcal{N}\left(0, a \theta+\sigma_{o}\right),
\end{aligned}
$$

where $\alpha$ is the terrestrial path-loss exponent, $a$ is the UAV shadowing slope, $\sigma_{o}$ is the UAV shadowing offset, $d$ denotes the ground distance between UAV and the serving BS (referred to Figure 1), $\theta$ is the depletion angle which approximately ranges from $\left(-2^{\circ}, 10^{\circ}\right), \theta_{o}$ is the angle offset, $A$ is the excess path-loss scaler and $B$ is the angle scaler 16 .

\section{SYSTEM PERFORMANCE ANALYSIS}

Assuming that all BSs except the serving BS cause interference to a UAV. The set of BSs that interfere the serving BS $X_{o}$ is $\Phi_{I}=\Phi \backslash\left\{X_{o}\right\}$. All interferers are located outside of the sphere $s\left(0, R_{o}\right)$, with $R_{o}$ is the sphere's radius, which is also the distance from a certain UAV located at the origin to the serving BS. Therefore, the aggregated interference at a certain UAV can be described as below:

$$
I=\sum_{\Phi_{I}} P_{o} g_{n} h_{n} L_{n}
$$

with $L_{n}$ is the path-loss contributing two random variables: the ground distance $d$ between
BS and UAV, and the depletion angle $\theta$. Those two random variables can be expressed in term of one random variable which is the distance $r$ as in Figure 1, )

Let $\left\{P_{n}\right\}_{n \in N^{+}}=P_{o} g h$ be a random variable vector with identical and independently distributed elements, the aggregated interference at a certain UAV can be rewritten as:

$$
I=\sum_{\Phi_{I}} P_{n} l\left(R_{n}\right)
$$

Let $W$ represent the Additive White Gaussian Noise (AWGN) power, the Signal-toInterference-plus-Noise-Ratio (SINR) at a UAV is

$$
S I N R=\frac{P_{R X}}{I+W},
$$

with $P_{R X}$ is the received power at UAV described in (1), I is the aggregated interference described in (4, 5).

Studying $m$ number of UAVs flying at the same height $h$. The optimal flying height and the system performance can be evaluated by finding the Cumulative Distribution Function (CDF) of the SINR in (6). The SINR's CDF can be found through computing the CDF of the aggregated interference I at a UAV in (5).

Since the CDF of the interference cannot be obtained in closed form [17, the CDF of the interference can be obtained by using the inverse Laplace transform [18]. With $s$ is the complex variable, Laplace transform for a random variable $X$ is defined as $\mathcal{L}_{X}(s)=\mathbb{E}\left[e^{-s X}\right]$. Hence, 
Laplace transform of the aggregated interference $I$ is described as below:

$$
\begin{aligned}
\mathcal{L}_{I}(s) & =\left[e^{-s I}\right] \\
& =\mathbb{E}_{\Phi_{I}} \mathbb{E}_{P}\left[e^{-s \sum_{\Phi_{I}} P_{n} l\left(R_{n}\right)}\right] \\
& =\mathbb{E}_{\Phi_{I}} \mathbb{E}_{P}\left[\prod_{\Phi_{I}} e^{-s P_{n} l\left(R_{n}\right)}\right] \\
& =\mathbb{E}_{\Phi_{I}}\left[\prod_{\Phi_{I}} \mathbb{E}_{P}\left[e^{-s P_{n} l\left(R_{n}\right)}\right]\right] \\
& =\mathbb{E}_{\Phi_{I}}\left[\prod_{\Phi_{I}} \mathcal{L}_{P}\left(-s l\left(R_{n}\right)\right)\right] .
\end{aligned}
$$

Let $F_{I}(x)$ denote the CDF of the interference I, $F_{I}(x)$ can be obtained through computing the inverse Laplace transform as

$$
\begin{aligned}
F_{I}(x) & =\mathcal{L}^{-1}\left[\frac{1}{s} \mathcal{L}_{I}(s)\right] \\
& =\mathcal{L}^{-1}\left[\frac{1}{s} \mathbb{E}_{\Phi_{I}}\left[\prod_{\Phi_{I}} \mathcal{L}_{P}\left(-s l\left(R_{n}\right)\right)\right]\right] \\
& =\mathcal{L}^{-1}\left[\frac{1}{s} \prod_{\Phi_{I}} \mathbb{E}_{\Phi_{I}}\left[\mathcal{L}_{P}\left(-s l\left(R_{n}\right)\right)\right]\right] \\
\mathrm{Using} E & {\left[\prod_{\Phi_{I}} f(x)\right]=e^{-2 \pi \lambda \int_{I}(1-f(x)) r d r}, \text { then } } \\
F_{I}(x) & =\mathcal{L}^{-1}\left[\frac{1}{s} e^{-2 \pi \lambda \int_{I}\left(1-e^{-s P_{n} l\left(R_{n}\right)}\right) r d r}\right] .
\end{aligned}
$$

When the SINR at a UAV falls below a threshold level $\tau$, the outage of service from serving BS will happen. Let $p_{S}$ denote the probability of successful reception at the UAV, $p_{S}$ is calculated as follows:

$$
\begin{aligned}
p_{S} & =\mathbb{E}_{R, h, g}\left[\mathbb{P}\left[S I N R \geq\left.\tau\right|_{h, g, R}\right]\right] \\
& =\mathbb{E}_{h, g}\left[\mathbb{E}_{R}\left[\mathbb{P}\left[S I N R \geq\left.\tau\right|_{h, g, R}\right]\right]\right] \\
& =\mathbb{E}_{h, g}\left[\mathbb{E}_{R}\left[\mathbb{P}\left[I \leq \frac{P_{R X}}{\tau}-W\right]\right]\right] \\
& =\mathbb{E}_{h, g}\left[\mathbb{E}_{R}\left[F_{I}\left(\frac{P_{R X}}{\tau}-W\right)\right]\right] \\
& =\mathbb{E}_{h, g}\left[\int_{r} F_{I}\left(\frac{P_{o} h g}{\tau r^{\alpha}}-W\right) .2 \lambda \pi r \exp \left(-\lambda \pi r^{2}\right) d r\right]
\end{aligned}
$$

with $F_{I}$ is the $\mathrm{CDF}$ of interference I calculated as in (8).
The system performance can be evaluated through the probability of successful reception $p_{S}$ in 10 . The optimized flying height can be obtained through optimizing random variable $R$.

\section{SIMULATION AND NUMERICAL RESULTS}

In this section, the numerical results for the network model will be validated. Simulation has

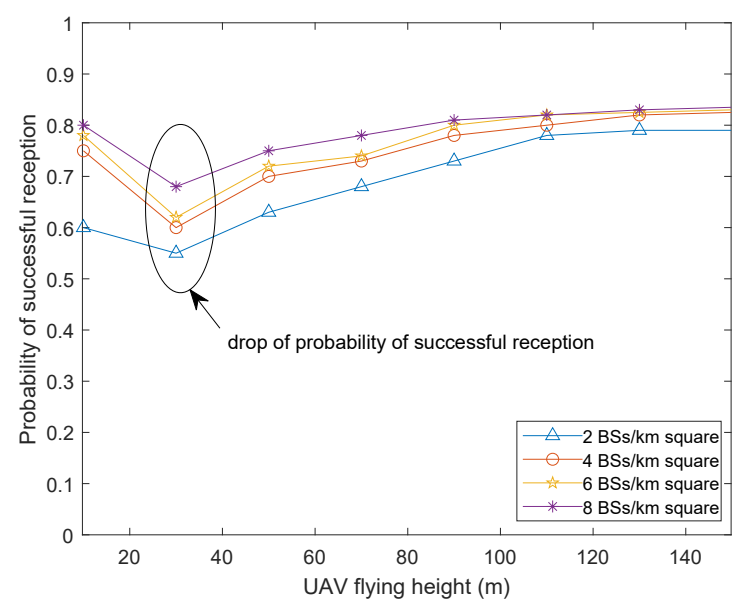

Fig. 2: UAV flying height to get successful reception from BSs with transmitted power of $50 \mathrm{~W}$.

been done for some cases that all UAVs operate at the same height in order to confirm the numerical results. The parameters used for numerical results and simulation are presented in Table 1. Note that all the parameters for path-loss are from experimental results and are adopted from [16]. The BSs using in the simulation are marco BSs with height of $30 \mathrm{~m}$ and transmitting power of $50 \mathrm{~W}$ (approximately 47 $\mathrm{dBm})$ (Figure 2) and $30 \mathrm{~W}$ (approximately 45 $\mathrm{dBm})$.

Figure 2 denotes the effect of UAV flying height on the system performance in term of the probability of successful reception at UAV side. These results are obtained with the default setup for the SINR threshold $\tau$ at the UAV antenna of $-8 \mathrm{~dB}$, transmitted power $P_{o}$ is $50 \mathrm{~W}$ in various BS density $\lambda$ condition. As shown in Figure 2 
Tab. 1: Network model parameters

\begin{tabular}{|l|l|r|}
\hline Parameter & Symbol & Value \\
\hline Terrestrial path-loss exponent & $\alpha$ & 3.04 \\
UAV shadowing slop & $a$ & -0.41 \\
UAV shadowing offset & $\sigma_{o}$ & 5.86 \\
Angle offset & $\theta_{o}$ & -3.61 \\
Excess path-loss scaller & $A$ & -23.29 \\
Angle scaler & $B$ & 4.14 \\
BS transmitted power & $P_{o}$ & $50 \mathrm{~W}$ \\
BS height & $h_{B S}$ & $30 \mathrm{~m}$ \\
Channel fading amplitude & $\alpha_{f}$ & 3 \\
Rice factor (Rayleigh fading \fading-less channel) & $K$ & $0 \backslash \infty$ \\
\hline
\end{tabular}

the successful reception probability under all BS densities drop when UAV flying height is under $30 \mathrm{~m}$. At this flying height, UAVs get reception from the main lobe of BS antenna and only terrestrial path-loss is considered. In this flying range, the probability of getting non-Line-ofSight is higher due to the blocking of infrastructure. In the higher range of flying height, the probability of successful reception is increased thanks to high probability of Line-of-Sight. In this flying height range, UAVs place themselves in the upper side lobes of BS antenna. The increase of BS density also slightly increase the probability of successful reception, however, it does not have much impact. Note that the flying height of UAV should not exceed $120 \mathrm{~m}$ according to the US Federal Aviation Administration guide for low attitude platform unmanned aerial vehicles defined as vehicles flying under 400 feet above the ground. Under the same condition, results in Figure 3 show the effect of transmitted power on the system performance. With higher transmitted power, we have better chance to get successful reception when UAVs fly higher.

Figure 4 shows the effect of SINR threshold $\tau$ at the UAV antenna on the probability of successful reception from BS. This results are collected under the scenario of having $4 \mathrm{BS}$ in a kilometer square with UAV flying height of 100 $\mathrm{m}$ and transmitted power of $50 \mathrm{~W}$. The path-loss considered includes terrestrial and aerial pathloss. As shown in Figure 4 with the increase of SINR threshold, the probability of successful reception decreases. This is simply due to the increase of interference at UAV side. The

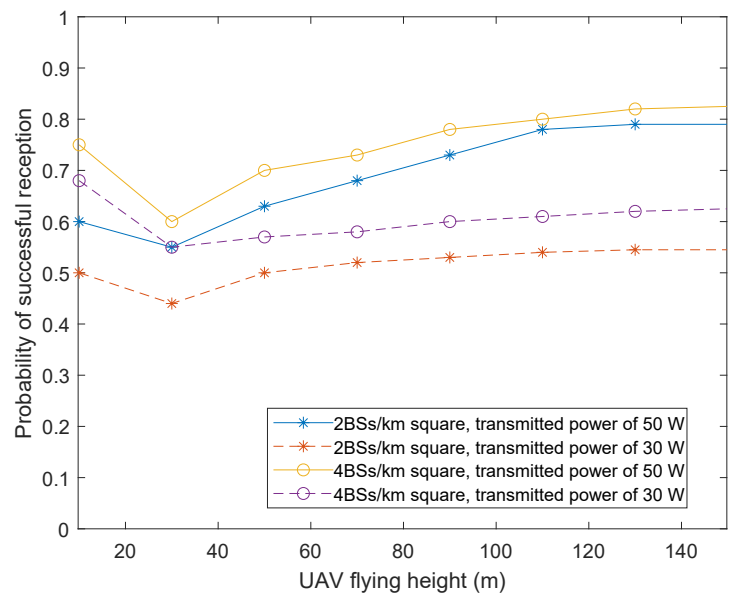

Fig. 3: UAV flying height to get successful reception with various transmitted power.

numerical results show that fading-less channel (path-loss only scenario in Figure 4) gives higher probability of successful reception than Rayleigh fading channel.

\section{CONCLUSIONS}

This paper uses stochastic geometry analysis to evaluate probability of getting cellular reception for UAV in FANET. With different base station density, the effect of UAV flying height on the network performance has been observed. Also for the network performance analysis, various parameters that directly impact the probability of successful reception have also been examined. With a predetermined SINR threshold, densed 


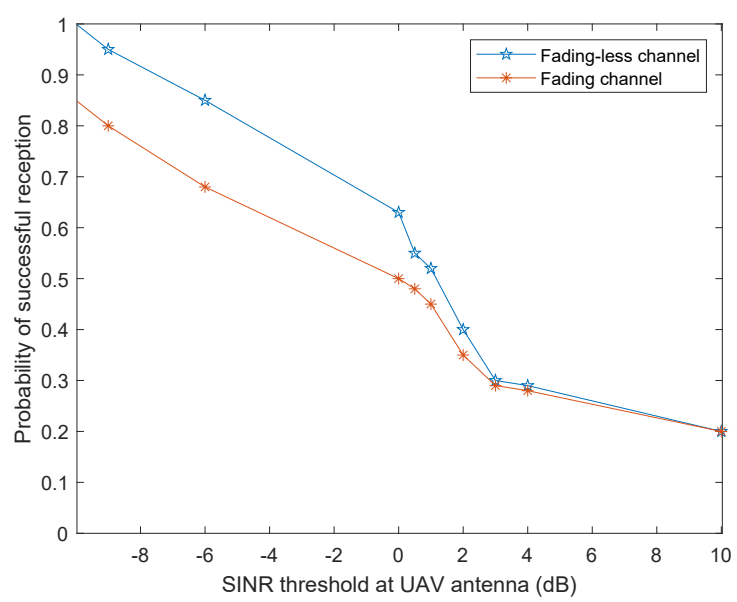

Fig. 4: The effect of SINR threshold at UAV side on the performance of the system.

BS scenarios give slightly higher chance of successful reception. The optimized flying range for UAV is above $100 \mathrm{~m}$ for best cellular reception. The SINR threshold at UAV antenna also has major impact on the system performance. Under fading-less channel, smaller SINR threshold gives better performance.

\section{References}

[1] A. Al-hourani, S. Kandeepan, A. Jamalipour, "Modeling air-to-ground path loss for low altitude platforms in urban environment," IEEE Global Telecommunications Conference (GLOBECOME), USA, 2014.

[2] A. Al-hourani, K. Sithamparanathan, "Optimal LAP altitude for maximum coverage," IEEE Wireless Communications Letters, vol. 3, no. 6, pp. 569-572, 2014.

[3] Y. Zheng, Y. Wang, F. Meng, "Modeling and simulation of path loss and fading for air-ground link of HAPs within a network simulator," Proc. of IEEE International Conference on Cyber-Enabled Distributed Computing and Knowledge Discovery (CyberC), China, 2013.

[4] D. Matolak, "Air-ground channels amp; models: Comprehensive review and con- siderations for unmanned aircraft systems," Proc. of IEEE Aerospace Conference, USA, 2012.

[5] K. Sasloglou, I. Glover, V. Gazis, et. al, "Empirical channel models for optimized communications in a network of unmanned ground vehicles," Proc. of IEEE International Symposium on Signal Processing and Information Technology, 2013.

[6] Y. Jung, Y. Kang, K. Son, H. W. Kim, K. Lim, "Air-ground channel model selection method for UAS communications using digital elevation data," 2017 Ninth International Conference on Ubiquitous and Future Networks (ICUFN), Milan, 2017.

[7] L. Zeng, X. Cheng, C. Wang, X. Yin, "A 3D geometry-based stochastic channel model for UAV-MIMO channels," 017 IEEE Wireless Communications and Networking Conference (WCNC), USA, 2017.

[8] M. Mozaffari, W. Saad, M. Bennis, M. Debbah, "Unmanned Aerial Vehicle with underlaid Device-to-Device communications: Performance and tradeoffs," IEEE Transactions on Wireless Communications, vol. 15, no. 6, pp. 3949-3963, 2016.

[9] A. Hayajneh, S. Zaidi, D. Mclernon, M. Ghogho, "Optimal dimensioning and performance analysis of drone-based wireless communications," Proc. of IEEE GLOBECOM Workshops, 2016.

[10] Q. Wu, Y. Zeng, R. Zhang, "Joint trajectory and communication design for multiuav enabled wireless networks," IEEE Transactions on Wireless Communications, Early access, 2018.

[11] H. Wang, G. Ren, J. Chen, G. Ding, Y. Yang, "Unmanned aerial vehicle-aided communications: Joint transmit power and trajectory optimization," IEEE Wireless Communications Letters, Early access, 2018.

[12] B. Der Bergh, A. Chiumento, S. Pollin, "LTE in the sky: trading off propagation benefits with interference costs for aerial nodes," IEEE Communications Magazine, vol. 54, no. 5, pp. 44-50, 2016. 
[13] M. Azari, F. Rosas, A. Chiumento, S. of Sciences, Vietnam. Currently, she is a Pollin, "Coexistence of terrestrial and $\mathrm{PhD}$ candidate of the School of Science and aerial users in cellular networks," Proc. of IEEE Global Telecommunications Conference (GLOBECOM) Workshops, Singapore, 2017.

[14] X. Lin, et.al, "The Sky Is Not the Limit: LTE for Unmanned Aerial Vehicles," IEEE Communications Magazine, vol. 56, no. 4, pp. 204-210, 2018.

[15] M. Tsai, "Path-loss and Shadowing (Largescale Fading)", Oct. 2011.

[16] A. Al-hourani, K. Gomez, "Modeling Cellular-to-UAV Path-loss for Suburban Environments," IEEE Wireless Communications Letters, vol. 7, no. 1, pp. 82-85, 2018 .

[17] M.z. Win, P.c. Pinto, L.a. Shepp, "A Mathematical Theory of Network Interference and Its Applications," Proceeding of the IEEE, vol. 97, no. 2, pp. 205-230, 2009.

[18] J. Abate, W. Whitt, "Numerical Inversion of Laplace Transforms of Probability Distributions," ORSA J. Comput., vol. 7, pp. 36-43, 1995.

\section{About Authors}

Quynh Tu NGO received her B.Eng. in Mechanical Engineering from HCM City University of Technology, Vietnam, in 2008. In 2013, she received her second B.Eng. in Electrical Engineering (Magna Cum Laude) from California State University Los Angeles, USA. In 2016, Quynh got her M.Sc. in Telecommunication Engineering from HCM City University Technology, Royal Melbourne Institute of Technology University, Australia. She has worked as a lecturer for Faculty of Electrical and Electronics Engineering, Ton Duc Thang University, Vietnam since 2014. Her research interests include medium access control for wireless networks (Adhoc, VANET, WSN...) and wireless communication.

Duc Ngoc Minh DANG is an Assistant Professor in Ton Duc Thang University, Vietnam. He received his B.Eng and M.Eng degrees in Telecommunications engineering from $\mathrm{Ho}$ Chi Minh City University of Technology, Vietnam, in 2005 and 2007, respectively. In 2014, he received the $\mathrm{PhD}$ degree in Computer engineering from Kyung Hee University, Korea. From 2005 to 2008, he was a Senior Telecom Engineer with TMA Solutions, Vietnam. Since 2008, he has worked as the Head of Electronics and Telecommunications Department and Vice Dean of School of Graduate Studies at Ton Duc Thang University, Vietnam. His research interests include MAC protocols in wireless ad hoc networks and vehicular ad hoc networks.

Khoa Anh TRAN became a lecturer for Faculty of Electrical and Electronic Engineering in Ton-Duc-Thang university, HCMC, Vietnam from 2010. He received the Ph.D. degrees in Telecommunication from the University of Siena, Siena, Italy, in 2017. His current research interests include Device-to-Device communication, V2I communication and Internet of Things.

"This is an Open Access article distributed under the terms of the Creative Commons Attribution License, which permits unrestricted use, distribution, and reproduction in any medium, provided the original work is properly cited (CC BY 4.0)." 